\title{
ESTUDO DA VARIAÇÃO DA MICROFILAREMIA EM CÃES INFESTADOS POR Dirofilaria immitis*
}

\author{
STUDY OF THE MICROFILAREMIC VARIATION IN DOGS \\ WITH Dirofilaria immitis
}

Maria Helena Matiko Akao LARSSON'; Marcia Cecília MENG²; Archivaldo RECHE JÚNIOR ; Carlos Eduardo LARSSON'; Masao IWASAKI ${ }^{4}$; Luiz Fernando DUTRA ${ }^{5}$

\begin{abstract}
RESUMO
Utilizaram-se 15 cães adultos, de ambos os sexos, com e sem definição racial, todos positivos para a presença de microfilárias na circulação periférica, para a observação da periodicidade das mesmas. A contagem das microfilárias foi realizada conforme ANGUS' (1981). A variação da microfilaremia foi observada durante um período de 24 horas, com amostras colhidas a intervalos de duas horas. Seis dos 15 cães foram submetidos a duas colheitas de material, uma em dia quente (temperatura ambiente média de $23^{\circ} \mathrm{C}$ ) e outra em dia frio (temperatura ambiente média de $16^{\circ} \mathrm{C}$ ). Analisando os resultados obtidos pode-se afirmar que as microfilárias de Dirofilaria immitis apresentam uma periodicidade, porém esta é muito variável, não obedecendo a um padrão fixo. Pode-se afirmar, também, que, uma vez presente, a microfilaremia não desaparece numa ou noutra colheita subseqüente, não dificultando o diagnóstico da parasitose.
\end{abstract}

UNITERMOS: Dirofilaria; Cães; Dirofilaria immitis; Microfilaria

\section{INTRODUÇÃO}

\begin{abstract}
A Dirofilaria immitisé um nematóide que parasita as cavidades cardíacas direitas e artérias pulmonares, acometendo, principalmente, os espécimes caninos. A dirofilariose canina pode manifestar-se de várias maneiras, sendo a mais freqüente a forma cardiopulmonar. Entretanto, independente da forma clínica do processo, o diagnóstico definitivo baseia-se na detecção de microfilárias no sangue periférico ${ }^{9}$.
\end{abstract}

MANSON" (1879) observou que as microfilárias de Wuchereria bancrofti, em pacientes infectados, eram numerosas durante a noite e mais raras, ou mesmo ausentes, durante o dia. Este fenômeno de alternância do número de microfilárias no decorrer de um período de 24 horas é denominado e conhecido como periodicidade das microfilárias. Segundo HAWKING ${ }^{6}(1960)$, a periodicidade das microfilárias está vinculada à transmissão da doença por artrópodes vetores; portanto, o período do aumento da concentração de microfilárias no sangue periférico coincide, habitualmente, com a hora do dia em que o vetor hematófago costuma se alimentar.

Na prática da Clínica Médica, tem-se observado, com certa freqüência, a ocorrência de casos de cães com sintomatologia compatível com dirofilariose, mas que

\footnotetext{
1- Professor Associado - Faculdade de Medicina Veterinária e Zootecnia da USP-SP

2 - Médico Veterinário - Faculdade de Medicina Veterinária e Zootecnia da USP-SP

3 - Pós-Graduando - Faculdade de Medicina Veterinária e Zootecnia da USP-SP

4 - Professor Titular - Faculdade de Medicina Veterinária e Zootecnia da USP-SP

5 - Médico Veterinário - São Paulo - SP

* Trabalho apresentado no XI Congresso Panamericano de Ciências Veterinárias realizado em Lima, Peru, 14 a 20 de agosto de 1988

Trabalho realizado sob os auspícios da FAPESP
}

não apresentam microfilaremia periférica ${ }^{2,9,18,21}$.

A pesquisa da literatura relativa ao assunto revela a existência de alguns trabalhos pertinentes à periodicidade das microfilárias $1,4,6,7,10,16$.

A maioria dos pesquisadores tem-se referido ao fato de que a concentração de microfilárias de Dirofilaria immitis é mais elevada à noite ${ }^{4,6}$, embora ${ }^{5,15}$, tenham observado picos diurnos de microfilaremia e, ainda, de acordo com OTTO et al. ${ }^{17}$ (1976), as microfilárias podem ser encontradas no sangue periférico várias vezes ao dia e a periodicidade das mesmas variar conforme a localização geográfica.

Outros trabalhos fazem crer que existam fatores adicionais que interferem na periodicidade das microfilárias, como a magnitude da microfilaremia (isto é, animais com microfilaremia elevada e moderada exibiriam pico maior às 16 horas e aqueles com microfilaremia leve apresentariam picos mais evidentes às 24 horas)' e a temperatura ambiente (isto é, a periodicidade das microfilárias de Dirofilaria immitis seria diretamente proporcional à temperatura ambiente) ${ }^{10}$. 
LARSSON, M.H.M.A.; MENG, M.C.; RECHE JÚNIOR, A.; LARSSON.C.E.; IW ASAKI, M.; DUTRA, L.F. Estudo da variação da microfilaremia em cães infestados por Dirnfilaria immitis. Braz. J. vet. Res. anim. Sci., São Paulo, v.32, n. 2, p. 1 14-9, 1995.

O escopo deste trabalho foi estudar a periodicidade das microfilárias de Dirofilaria immitis em cães de nosso meio; em caso positivo, ou seja, de existir(em) realmente pico(s) de microfilaremia, caracterizá-lo(s) com a finalidade de indicar $\mathrm{o}(\mathrm{s})$ período(s) do dia mais propício(s) para a colheita de sangue de animais suspeitos, aumentando assim as possibilidades diagnósticas. Pretendeu-se também correlacionar a(s) periodicidade(s) a variações de temperatura ambiente.

\section{MATERIAL E MÉTODO}

O grupo experimental foi constituido por 15 animais adultos, de ambos os sexos, com e sem definição racial, procedentes dos municipios paulistas de Guarujá, Bertioga e Cubatão.

Os animais incluídos no presente trabalho foram selecionados por meio da pesquisa de microfilárias circulantes [Método da Gota Espessa; Técnica de KNOTT modificada e Técnica de MELLO; $\operatorname{MAUGE}^{13}$ (1983)] e/ou manifestações clínicas e/ ou exames complementares como exames radiográfico e eletrocardiográfico.

Para a observação da periodicidade das microfilárias circulantes utilizou-se a contagem das mesmas, segundo $\operatorname{ANGUS}^{1}$ (1981).

Exceção feita a três animais (domiciliados), os demais receberam tratamento anti-helmíntico (Mebendazole, na dose de $30 \mathrm{mg} / \mathrm{kg} /$ dia, durante três dias) e foram vacinados contra Cinomose, Hepatite Infecciosa Canina, Leptospirose e Parvovirose, respectivamente, 40 e 30 dias antes do início do experimento.

Durante todo o período de experimentação, os animais não domiciliados foram mantidos em canis individuais do Departamento de Clínica Médica da Faculdade de Medicina Veterinária e Zootecnia, recebendo, diariamente, ração comercial**. $^{* *}$.

\section{Colheita de Material}

O material (sangue, na quantidade de $3 \mathrm{ml}$ ) destinado à pesquisa e contagem de microfilárias foi obtido por punção das veias radiais e/ou safenas e/ou jugulares, a intervalos de duas horas, durante um periodo de 24 horas, e acondicionado em frascos contendo $0,03 \mathrm{ml}$ de EDTA (sal sódico do ácido etilenodiaminotetracético a $10 \%$ ) para posterior análise.

A colheita de material dos animais (domiciliados) foi realizada numa clínica particular, na cidade de Guarujá, ao passo que o material referente aos demais foi colhido nos próprios do
Departamento de Clínica Médica da FMVZ/USP.

Seis cães (noي $3,4,5,6,7$ e 8 ) foram submetidos a duas colheitas de material, uma em dia quente (temperatura ambiente média de $23^{\circ} \mathrm{C}$ ) e outra em dia frio (temperatura ambiente média de $16^{\circ} \mathrm{C}$ ).

\section{Método de Gota Espessa}

Uma gota de sangue com anticoagulante é examinada entre lâmina e lamínula; as larvas são vistas, neste método, indiretamente pela movimentação das hemácias. As microfilárias de Dirofilaria immitis são dotadas de movimentos serpentiformes?.

\section{Técnica de KNOTT modificada}

Consiste na técnica padrão (NEWTON; WRIGHT $\left.{ }^{14}, 1956\right)$ é processada da seguinte forma: $1 \mathrm{ml}$ de sangue é hemolisado em $10 \mathrm{ml}$ de formol $2 \%$; após centrifugação por cinco minutos a 1500 rpm, o sedimento é misturado com igual quantidade de Azul de Metileno (1:1000) e examinado ao microscópio óptico para observação, principalmente, das características morfológicas das microfilárias.

\section{Técnica de MELLO; MAUGE ${ }^{13}$ (1983)}

- Colocam-se, em tubo de centrífuga, 8-10 $\mathrm{ml}$ da seguinte solução:

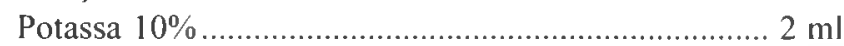

Detergente comercial ........................................ $2 \mathrm{ml}$

Água destilada .............................................. $1000 \mathrm{ml}$

adicionar 0,2 $\mathrm{ml}$ de sangue e hemolisar;

- centrifuga-se a 3000 rpm durante 10 minutos;

- adicionam-se uma a duas gotas de glicerina corada pelo Azul de Metileno (glicerina $100 \mathrm{ml}+$ Azul de Metileno 200 $\mathrm{mg}$ ); haverá deslocamento da gota de glicerina para o fundo do tubo;

- centrifuga-se novamente por cinco minutos a 3000 rpm;

- retira-se o sobrenadante até o nível superior da gota de glicerina corada, com o auxílio de bomba de vácuo (trompa d'água), ligada a uma torneira de água corrente. O tubo plástico da extremidade da bomba deve ter aberturas laterais para evitar a sucção do sedimento;

- retira-se todo o sedimento com o auxílio de uma pipeta Pasteur e coloca-se entre lâmina e lamínula para ser feita a observação.

\footnotetext{
** Gentilmente cedida pela Mogiana Alimentos.
} 
LARSSON, M.H.M.A.; MENG, M.C.; RECHE JÚNIOR, A.; LARSSON, C.E.; IWASAKI, M.; DUTRA, L.F. Estudo da variação da microfilaremia em cães infestados por Dirofilaria immitis. Braz. J. vet. Res. anim. Sci., São Paulo, v.32, n.2, p.114-9, 1995.

\section{Interpretação}

Para a diferenciação das microfilárias podem-se levar em conta as seguintes características morfológicas:

\section{D. immitis D. reconditum}

Extremidade posterior em gancho ausente presente Presença de gancho cefälico ausente presente

Estas características morfológicas tornam-se bastante visíveis quando coradas pelo Azul de Metileno.

Método para contagem de microfilárias (ANGUS', 1981)

Dilui-se $1 \mathrm{ml}$ de sangue em $6,6 \mathrm{ml}$ de líquido estromalizante ( $8 \mathrm{~g}$ de bicarbonato de sódio; $5 \mathrm{ml}$ de Triton detergente e água destilada qsq $1000 \mathrm{ml}$ ). Preenche-se a câmara de McMaster com mistura sangue-líquido estromalizante e contam-se as microfilárias em aumento de 100 vezes.

O resultado final, isto é, o número de microfilárias por $\mathrm{ml}$ de sangue, é obtido multiplicando-se o número de parasitas pelo fator 50,66 :

Área da câmara de McMaster $0,15 \mathrm{ml}$

Diluição do sangue no fluido $1: 7,6$

Área de sangue na câmara $7,6: 0,15=50,66$

\section{Manifestações Clínicas}

As manifestações clínicas consideradas, para a triagem dos animais domiciliados, foram as que se seguem: intolerância ao exercício, tosse, dispnéia, ascite e edema subcutâneo. Obviamente, os animais também foram submetidos aos exames laboratoriais para a pesquisa de microfilárias circulantes.

\section{Exames Radiográfico e Eletrocardiográfico}

O animal no 3 (domiciliado) foi avaliado também por meio de exames radiográfico e eletrocardiográfico.

Oexame radiográfico foi realizado na disciplina de Radiologia do Departamento de Cirurgia e Obstetrícia da Faculdade de Medicina Veterinária e Zootecnia da Universidade de São Paulo. As radiografias foram efetuadas em aparelhos de raios$\mathrm{X}$, modelo Tridoros 4, de capacidade para $1000 \mathrm{~mA}$, equipado com grade antidifusora Potter-Bucky***.

A técnica radiográfica empregada foi a que relaciona miliamperagem-segundo e quilovoltagem à espessura da região torácica ${ }^{3}$.
O traçado eletrocardiográfico foi realizado com os animais, sem anestesia, mantidos em decúbito lateral direito; registraram-se as derivações bipolares I, II, III e as unipolares aumentadas aVR, aVF, aVL ${ }^{8,20}$. Utilizou-se eletrocardiógrafo FUNBEC modelo ECG-3.

\section{RESULTADOS}

Os resultados obtidos acham-se dispostos sob a forma de tabelas (Tab. 1, 2).

Ainda, para uma melhor visualização dos resultados, dispuseram-se na Fig. I os representados pelas médias, em diferentes tempos de colheita de sangue.

\section{COMENTÁRIOS}

Analisando os resultados obtidos no presente trabalho e conceituando periodicidade, segundo SASA; TANAKA ${ }^{19}$ (1972), como "uma elevação e diminuição cíclicas do número de microfilárias no decorrer do tempo", pode-se afirmar que os animais naturalmente infectados por Dirofilaria immitis ¿p. esentam periodicidade de microfilárias; porém, esta periodicidade é muito variável de animal para animal, não obedecendo a um padrão fixo.

Ao contrário do que foi afirmado por $\mathrm{HAWKING}^{6}(1960) \mathrm{e}$ por GLUBER ${ }^{4}$ (1966), não se observou periodicidade noturna marcante na maioria dos animais. Nossos resultados também discordam daquele de $\mathrm{KUME}^{10}$ (1974), que referiu ser o número de microfilárias circulantes diretamente proporcional à temperatura ambiente.

O que se pode afirmaré que, uma vez presente, a microfilaremia não desaparece numa ou noutra colheita subseqüente, facilitando assim o diagnóstico da parasitose cardíaca. Se ocorrer microfilaremia negativa em associação com manifestações compatíveis com dirofilariose deve-se pensar na possibilidade de se tratar de um caso oculto da referida parasitose, não havendo necessidade de preconizar novas colheitas de sangue, em horários variados, para a detecção das microfilárias na circulação.

Nossos resultados são parcialmente semelhantes àqueles obtidos por ANGUS ${ }^{1}$ (1981), que afirma que as microfilárias de Dirofilaria immitis podem ser encontradas no sangue periférico em várias ocasiões, durante todo o dia. No presente trabalho, de 15 animais estudados, alguns com duas colheitas, totalizando 21 colheitas durante as 24 horas do dia, observamos picos de contagem, ora noturnos, ora diurnos, 
LARSSON, M.H.M.A.; MENG, M.C.; RECHEJÚNIOR, A.; LARSSON,C.E.; IWASAKI, M.; DUTRA, L.F. Estudoda variaçãoda microfilaremia em cāes infestados porDirofilaria immitis. Braz. J. vet. Res. anim. Sci., São Paulo, v.32, n.2, p.114-9, 1995

TABELA 1

Contagem do número de microfilárias de 15 cães segundo horário de colheita e temperatura ambiente. São Paulo, 1988.

\begin{tabular}{|c|c|c|c|c|c|c|c|c|c|c|c|c|c|}
\hline \multicolumn{14}{|c|}{ Número de microfilárias de Dirofilaria immitis no sangue periférico } \\
\hline $\begin{array}{c}\text { Animal } \\
n^{0}\end{array}$ & $\begin{array}{l}\text { Tempo } \\
\text { Colheita }\end{array}$ & $2 \mathrm{~h}$ & $4 \mathrm{~h}$ & $6 \mathrm{~h}$ & $8 \mathrm{~h}$ & $10 \mathrm{~h}$ & $12 \mathrm{~h}$ & $14 \mathrm{~h}$ & $16 \mathrm{~h}$ & $18 \mathrm{~h}$ & $20 \mathrm{~h}$ & $22 \mathrm{~h}$ & $24 \mathrm{~h}$ \\
\hline 1 & A & 30.258 & 23.883 & 43.566 & 40.378 & 39.012 & 34.964 & 55.052 & 41.694 & 37.342 & 31.119 & 57.835 & 43.617 \\
\hline 2 & A & 15.180 & 11.992 & 16.495 & 12.397 & 9.968 & 16.040 & 13.459 & 15.281 & 18.772 & 12.650 & 16.799 & 13.662 \\
\hline 3 & A & 115.671 & 156.961 & 881.972 & 93.002 & 171.331 & 57.886 & 29.955 & 83.287 & 198.048 & I33.584 & 81.769 & 199.667 \\
\hline 4 & A & 1.669 & 2.074 & 5.262 & 3.643 & 4.452 & 8.045 & 4.452 & 4.098 & 2.479 & 5.060 & 2.530 & 2.125 \\
\hline 5 & A & 3.643 & 1.214 & 22.125 & 2.277 & 2.125 & 1.518 & 2.175 & 1.771 & 2.833 & 2.327 & 3.440 & 2.833 \\
\hline 6 & A & 910 & 2.024 & 1.113 & 4.048 & 3.187 & 2.327 & 2.277 & 3.137 & 3.542 & 2.327 & 1.619 & 1.922 \\
\hline 7 & $\mathrm{~A}$ & 6.628 & 11.486 & 13.813 & 18.924 & 24.035 & 11.638 & 15.382 & 18.418 & 9.259 & 14.168 & 8.096 & 16.596 \\
\hline 8 & A & 27.981 & 26.818 & 23.984 & 29.904 & 34.610 & 22.264 & 46.552 & 28.639 & 37.089 & 25.401 & 19.076 & 31.878 \\
\hline 9 & $\mathrm{~A}$ & 24.841 & 17.982 & 20.845 & 24.109 & 19.047 & 29.637 & 40.492 & 16.050 & 28.238 & 27.639 & 25.308 & 20.179 \\
\hline 10 & A & 88.711 & 54.412 & 62.937 & 62.470 & 62.271 & 26.709 & 51.548 & 66.067 & 28.704 & 75.591 & 64.069 & 45.487 \\
\hline 11 & $A$ & 13.386 & 22.377 & 13.519 & 9.257 & 15.251 & 12.720 & 8.458 & 8.258 & 9.457 & 8.458 & 17.049 & 8.591 \\
\hline 12 & $\mathrm{~A}$ & 23.243 & 20.579 & 9.457 & 18.914 & 14.052 & 11.521 & 14.585 & 16.317 & 19.047 & 21.045 & 32.634 & 15.251 \\
\hline 13 & A & 10.456 & 6.726 & 8.658 & 6.793 & 15.318 & 8.325 & 8.391 & 7.525 & 10.989 & 11.854 & 11.388 & 8.924 \\
\hline 14 & A & 42.424 & 24.175 & 17.982 & 35.431 & 29.304 & 15.138 & 13.986 & 18.914 & 23.367 & 23.709 & 30.169 & 28.638 \\
\hline 15 & A & 1.065 & 1.065 & 1.198 & 1.598 & 1.531 & 1.598 & 1.665 & 1.332 & 999 & 1.665 & 666 & 666 \\
\hline \multicolumn{2}{|l|}{ Média } & 27.071 & 25.585 & 76.195 & 24.210 & 29.700 & 17.355 & 20.562 & 22.053 & 28.678 & 26.440 & 24.830 & 29.336 \\
\hline \multicolumn{2}{|c|}{ Desvio Padrão } & 32.139 & 37.623 & 215.918 & 24.712 & 41.086 & 14.613 & 18.349 & 23.347 & 46.807 & 33.732 & 24.024 & 47.636 \\
\hline
\end{tabular}

$\mathrm{A}=$ Dias quentes

TABELA 2

Contagem do número de microfilárias de seis cães segundo horário de colheita e temperatura ambiente. São Paulo, 1988.

Número de microfilárias de Dirofilaria immitis no sangue periférico

\begin{tabular}{|c|c|c|c|c|c|c|c|c|c|c|c|c|c|}
\hline $\begin{array}{c}\text { Animal } \\
\mathrm{n}^{\mathrm{g}}\end{array}$ & $\begin{array}{l}\text { Tempo } \\
\text { Colheita }\end{array}$ & $2 \mathrm{~h}$ & $4 \mathrm{~h}$ & $6 \mathrm{~h}$ & $8 \mathrm{~h}$ & $10 \mathrm{~h}$ & $12 \mathrm{~h}$ & $14 \mathrm{~h}$ & $16 \mathrm{~h}$ & $18 \mathrm{~h}$ & $20 \mathrm{~h}$ & $22 \mathrm{~h}$ & $24 \mathrm{~h}$ \\
\hline 3 & B & 101.706 & 100.997 & 91.484 & 101.351 & 106.715 & 60.770 & 110.662 & 144.564 & 75.596 & 95.026 & 101.908 & 69.828 \\
\hline 4 & B & 3.744 & 1.315 & 6.426 & 5.060 & 3.592 & 2.226 & 2.125 & 2.074 & 1.214 & 2.732 & 1.518 & 1.922 \\
\hline 5 & B & 1.720 & 1.771 & 1.315 & 1.821 & 1.973 & 1.467 & 1.416 & 1.872 & 354 & 1.016 & 1.315 & 1.568 \\
\hline 6 & B & 2.378 & 3.440 & 2.681 & 2.175 & 8.956 & 1.669 & 1.366 & 2.277 & 2.074 & 2.428 & 2.428 & 2.226 \\
\hline 7 & B & 8.555 & 13.156 & 9.917 & 7.843 & 8.096 & 6.021 & 9.006 & 8.804 & 13.156 & 13.004 & 5.161 & 5.414 \\
\hline 8 & B & 31.827 & 37.646 & 49.486 & 31.878 & 17.204 & 24.186 & 17.001 & 56.064 & 73.117 & 23.377 & 34.711 & 29.246 \\
\hline Média & & 24.988 & 26.388 & 26.885 & 25.021 & 24.423 & 16.057 & 23.596 & 35.943 & 27.585 & 22.931 & 24.507 & 18.367 \\
\hline Desvio & Padrão & 35.838 & 35.659 & 33.241 & 35.653 & 37.121 & 21.514 & 39.336 & 52.251 & 33.352 & 33.179 & 36.568 & 24.992 \\
\hline
\end{tabular}

$\mathrm{B}=$ Dias frios 
LARSSON, M.H.M.A.; MENG, M.C.; RECHE JÚNIOR, A.; LARSSON,C.E.; IWASAKI, M.; DUTRA, L.F. Estudo da variação da microfilaremia em cāes infestados porDirofilaria immitis. Braz. J. vet. Res. anim. Sei., São Paulo, v.32, n.2, p.114-9, 1995.

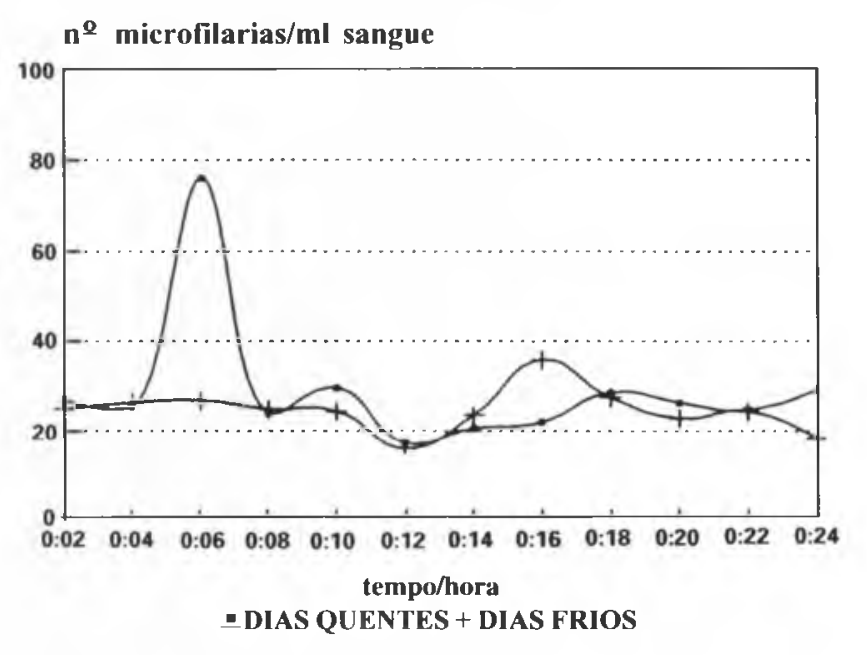

FIGURA 1

Periodicidade das microfilarias do Dirofilaria immitis no sangue periférico de 15 cães. São Paulo, 1988. mas em nenhuma ocasião as microfilárias deixaram de ser detectadas no período de observação de 24 horas. Observação semelhante à nossa foi feita por MATOLA ${ }^{12}$ (1991), que estudou a periodicidade das microfilárias de Dirofilaria im mitis durante cinco dias, realizando colheitas de hora em hora em um único animal.

\section{SUMMARY}

The periodicity of Dirofilaria immitis microfilariae was studied in 15 adult dogs, purebred or crossbred, males and females. The circulating microfilariae counting was performed according to ANC'LS' (1981). The variation of the microfilaremia was observed during a period of 24 hours, being the blood samples collected with 2 hour intervals. Six of them were submitted to two material collections, one on a warm day, with mean temperature of $23^{\circ} \mathrm{C}$, and the other one on a cool day, with mean temperature of $16^{\circ} \mathrm{C}$. Analyzing the obtained results it is possible to state that the microfilariae of Dirofilaria immitis present an oscillation, however it is variable and there is not a fixed pattern. It is also true that once present the microfilaremia does not disappear in a subsequent collection, making it not difficult to diagnose this parasitosis.

UNITERMS: Dirofilaria; Dogs; Dirofilaria immitis; Microfilariae

\section{REFERÊNCIAS BIBLIOGRÁFICAS}

1-ANGUS, B.W. Periodicity exhibited by microfilariae of D. immitis in Southeast Queensland. Australian Veterinary Journal, v.57, p.101-2, 1981.

2-CASTLEMAN, W.L.K.; WONG, M.M. Light and electron microscopic lesions associated with retained microfilariae in canine occult dirofilariosis. Veterinary Pathology, v.19, p.355-64, 1982.

3-DE MARTIN, B.W.; IWASAKI, M. Noções de radiodiagnóstico veterinário. São Paulo, 1976.

4 - GLUBER, D.J. A comparative study on the distribution, incidence and periodicity of the canine filarial worms Dirofilaria immitis Leydi and Dipetalonema reconditum Grassi in Hawaii.Journal of Medical Entomology, v.3, p.159$67,1966$.

5-GRIEVE, R.B.; LAURIA,S. Periodicity of Dirofilaria immitis microfilariae in canine and murine hosts.Acta Tropica. v. 40, p. $121-7,1983$

6-HAWKING, F. Periodicity of microfilariae.Indian Journal of Malariology, v.14, p.567-73, 1960.

7-HAWKING, F.; THURSTON, J.P. The periodicity of microfilariae. I. Distribution of microfilariae in the body. Transactions of the Royal Society of Tropical Medicine and Hygiene, v.45, p.307-28, 1951.

8-HILL, J.D. Electrocardiographic diagnosis of right ventricular enlargement in dogs. Journal of Electrocardiclogy, v.4, p.347-57, 1971 .

9-KNIGHT, D.H. Heartworm heart disease. Advances in Veterinary Science, v.21, p.107-47, 1971. 
LARSSON, M.H.M.A.; MENG, M.C.; RECHE JÚNIOR, A.; LARSSON, C.E.; IWASAKI, M.; DUTRA, L.F. Estudoda variação da microfilaremia em cães infestados porDirofilaria immitis. Braz. J. vet. Res. anim. Sci., São Paulo, v. 32, n.2, p. 114-9, 1995.

10-KUME, S. Experimental observations on seasonal periodicity of microfilariae. In: HEARTWORM SYMPOSIUM, 74, Proceedings, 1974. p.26-31.

11-MANSON, 1879 apud HAWKING, F. ., 1960. p. 567.

12-MATOLA, Y.G. Periodicity of Dirofilaria immitis microfilariae in a dog from Muheza district, Tanzania. Journal of Helminthology, v.65, p.76-8, 1991.

13-MELLO, E.B.F.; MAUGE, G.C. Nova técnica para diagnóstico e contagem de microfilárias. In: CONGRESSO DA FEDERACIÓN LATINO AMERICANA DE PARASITÓlOGOS. 6., São Paulo, 1983. Anais. p. 115.

14-NEWTON, W.L.; WRIGHT, W.H. The occurrence of a dog filariid other than Dirofilaria immitis in the United States. Journal of Parasitology, v.42, p.246-58, 1956.

15-NEWTON, W.L.; WRIGHT, W.H. A reevaluation of the canine filariasis problem in the United States. Veterinary Medicine, v.52, p.75-8, 1957.

16-OTTO, G.F.; JACKSON, R.F. Heartworm disease. In: ETTINGER, S.J. Texbook of veterinary internal medicine: diseases of dog and cat. 2. ed. Philadelphia, W.B. Saunders, 1975. v.2, p.1014-38.

17-OTTO, G.F.; JACKSON, R.F.; BAWMAN,P.M.; PEA-
COCK, F.; RICHS, W.L.; MAITBY, J.H. Variability in the ratio between the numbers of microfilariae and adult heartworms. Journal of the American Veterinary Medical Association, v.168, p.605-7, 1976.

18-RAWLINGS,C.A.; DAWE,D.L.; McCALL,J.W.;KEITH, J.C.; PRESTWOOD, A.K. Four types of occult Dirofilaria immitis infection in dogs. Journal of the American Veterinary Medical Association, v.180, p. 123-61, 1982.

19-SASA, M.; TANAKA, H. Studies on the methods for statistical analysis of the microfilarial periodicity survey data. Southest Asian Journal of Tropical Medicine and Public Health, v.4, p.518-36, 1972.

20-TILLEY, L.P. Essentials of canine and feline electrocardiography. Saint Louis, C.V. Mosby, 1979.

21-WONG, M.M.; SUTER, P.F.; THODE, E.A.; GUEST, M.F. Dirofilariasis without circulating microfilariae: a problem in diagnosis.Journal of the American Veterinary Medical Association, v.163, p.133-9, 1973.

Recebido para publicação em 17/07/93 Aprovado para publicação em 07/11/94 